APPLIED MATHEMATICS AND MECHANICS (ENGLISH EDITION)

https://doi.org/10.1007/s10483-020-2676-5

\title{
Size-dependent thermoelastic initially stressed micro-beam due to a varying temperature in the light of the modified couple stress theory*
}

\author{
A. E. ABOUELREGAL ${ }^{1,2, \dagger}$ \\ 1. Department of Mathematics, College of Science and Arts, Jouf University, \\ Al-Qurayyat 77423, Saudi Arabia; \\ 2. Department of Mathematics, Faculty of Science, Mansoura University, \\ Mansoura 35516, Egypt \\ (Received Mar. 24, 2020 / Revised Aug. 3, 2020)
}

\begin{abstract}
The bending of the Euler-Bernoulli micro-beam has been extensively modeled based on the modified couple stress (MCS) theory. Although many models have been incorporated into the literature, there is still room for introducing an improved model in this context. In this work, we investigate the thermoelastic vibration of a micro-beam exposed to a varying temperature due to the application of the initial stress employing the MCS theory and generalized thermoelasticity. The MCS theory is used to investigate the material length scale effects. Using the Laplace transform, the temperature, deflection, displacement, flexure moment, and stress field variables of the micro-beam are derived. The effects of the temperature pulse and couple stress on the field distributions of the micro-beam are obtained numerically and graphically introduced. The numerical results indicate that the temperature pulse and couple stress have a significant effect on all field variables.
\end{abstract}

Key words thermoelectricity, micro-beam, initial stress, temperature pulse, modified couple stress (MCS) theory

Chinese Library Classification O321, O327, O343

2010 Mathematics Subject Classification 74H45, 70J10, 74B10

\section{Introduction}

More recently, small scale and nano-scale structures have been used in several design devices such as nuclear drive and switches. A few models are developed for small scale and nanostructures, including strain gradient and couple stress. The modified couple stress (MCS) theory can be seen as an exceptional example of the strain gradient theory. The MCS theory considers the revolution as a variable in determining curvature, while the strain inclination theory considers the strain to be variable in curvature pronounce ${ }^{[1-4]}$.

The classical elasticity theory is not suitable to capture the effect of the size of the microstructure. In addition, it is appropriate to study the material behavior on a large scale. As

* Citation: ABOUELREGAL, A. E. Size-dependent thermoelastic initially stressed micro-beam due to a varying temperature in the light of the modified couple stress theory. Applied Mathematics and Mechanics (English Edition), 41(12), 1805-1820 (2020) https://doi.org/10.1007/s10483-020-2676-5

$\dagger$ Corresponding author, E-mail: ahabogal@gmail.com

(C)The Author(s) 2020 
the size of the study decreases, the accuracy of the classical theory diminishes and therefore its expectation of the material behavior on the micro- and nano-scales does not match with experimental results. It is observed that the explanation behind this deviation is the critical effect of the micro-structure ${ }^{[5-7]}$.

On the other hand, non-classical theories (e.g., strain gradient and couple stress) are used to concentrate on the material behavior on these scales. These theories also contain size scale factors which show the influence of micro-structure. The MCS model is noted to be the simplest among these models. Constitutive equations established for an isotropic material based on the classical MCS model include Lame's coefficients and two size scale factors.

Through the experimental observations of micro-/nano-scale structures, it is shown that mechanical properties depend on the size, and these properties cannot be seen when we use the classical theories of elasticity ${ }^{[8-11]}$. Hence, modified elasticity theories such as the MCS, the nonlocal continuum mechanics, the nonlocal strain gradient theory, and the strain gradient theory are widely used to analyze the dynamic and static responses of micro-/nano-scale structures $^{[12]}$.

An extensive modification of the classical MCS theory has been conducted and mathematically analyzed in Refs. [13]-[15]. In Ref. [16], the authors introduced the MCS model where the couple stress tensor was antisymmetric. However, the constitutive equations established by this theory consist of only one length scale factor. In Ref. [17], the reaction of the thermoelastic micro-beam using the MCS theory due to thermal source was introduced. In Ref. [18], the authors considered the beam problem using the Euler-Bernoulli model and the MCS theory.

More recently, higher-order continuum theories have been developed to predict the relationships dependent on the size-dependent size ${ }^{[19]}$. In the $1960 \mathrm{~s}$, some researchers proposed a couple stress theory of elasticity that could be classified as a non-classical theory ${ }^{[20]}$. The theory made it possible to explain the size-dependent effects using two higher-order material constants in state equations. A simplification introduced by Yang et al. ${ }^{[21]}$ to the scaling complex relations of the couple stress theory of elasticity resulted in the modified coupled stress theory which was suitable due to the effects using only one scale parameter for the length of the material. Recently, many researchers have investigated non-classical continuum theorems to formulate and study the size-dependent mechanical behavior of beams and plates.

Tsiatas $^{[22]}$ proposed a new model of Kirchhoff plates based on the MCS theory with the consideration of the size-dependent effects. Wang et al. ${ }^{[23]}$ proposed the Euler-Bernoulli beam model on the basis of the MCS model, and studied the effects of the length material parameter on the properties of the static mechanical micro-beam. Duan et al. ${ }^{[24]}$ used the Hamilton principle and relations of the non-local Eringen's theory to develop the non-local theories of the Euler-Bernoulli, Timoshenko, Reddy, and Levinson beams. An advanced continuum mechanics theory should be used to capture the size effects, and these papers used the MCS theory ${ }^{[25-28]}$.

This article contributes to introducing the thermomechanical response of micro-beams based on the generalized theory of thermoelasticity. The MCS theory is introduced to study the effect of size on small-scale Euler-Bernoulli beams. The micro-beam is subject to a varying temperature plus an initial compression stress. Using a combination of the dynamic equation of the transverse deflection, MCS theory and generalized thermoelasticity, two partial differential equations are derived and completely solved using the Laplace transform method. The effects of the scale parameter, initial stress, relaxation time, and pulse width on the temperature, stress, displacement, and couple stress are studied. The results of some previous work are also inferred from the current analysis as special cases. The current work is a framework for the models conducted in Refs. [29]-[31]. 


\section{Basic equations}

For simplicity and readability propose, all the mathematical notations appearing in this paper are defined in nomenclature. The basic equations of the isotropic homogenous thermoelastic medium (in the $O x y z$ coordinates) at an initial uniform temperature $T_{0}$ based on the MSC theory are given as follows ${ }^{[17,23,32]}$ :

$$
\begin{aligned}
& \sigma_{i j}=\lambda e_{k k} \delta_{i j}+2 \mu e_{i j}-\gamma \theta \delta_{i j}, \\
& m_{i j}=2 \alpha \chi_{i j}, \\
& \chi_{k l}=\frac{1}{2}\left(\frac{\partial \omega_{k}}{\partial x_{l}}+\frac{\partial \omega_{l}}{\partial x_{k}}\right), \\
& \omega_{i}=\frac{1}{2} e_{i j k} u_{k, j} \\
& e_{k l}=\frac{1}{2}\left(\frac{\partial u_{k}}{\partial x_{l}}+\frac{\partial u_{l}}{\partial x_{k}}\right),
\end{aligned}
$$

where $\lambda$ and $\mu$ denote Lame's constants, $\sigma_{i j}$ are components of the stress tensor, $e_{k l}$ are components of the strain tensor, $u_{k}$ is the displacement vector, $\theta=T-T_{0}$ is the excess temperature distribution, $\omega_{i}$ is the rotation vector, $m_{i j}$ is the couple stress tensor, $\alpha=l^{2} \mu$ is a higherorder modulus and is considered as the rotational modulus indicating the resistance of the material versus the gradient of its element rotation, $l$ is the material length scale parameter, $\gamma=\alpha_{\mathrm{t}} E /(1-2 \nu), E$ is Young's modulus, $\alpha_{\mathrm{t}}$ is the thermal expansion coefficient, $\nu$ is Poisson's ratio, and $\delta_{i j}$ is the Kronecker delta function. The parameter $\alpha$ is indeed a higher-order modulus which can be regarded as the rotational modulus representing the resistance of the material against the gradient of the rotation of its elements.

In this paper, we take the equation of heat conduction corresponding to that conducted in Ref. [33]. This equation can be written as follows:

$$
\nabla(K \nabla \theta)=\left(1+\tau_{0} \frac{\partial}{\partial t}\right)\left(\frac{K}{k} \frac{\partial \theta}{\partial t}+\gamma T_{0} \frac{\partial e_{k k}}{\partial t}\right),
$$

where $K$ is the thermal conductivity, $k=K /\left(\rho C_{E}\right), C_{E}$ is the specific heat per unit mass at constant strain, $\tau_{0}$ indicates the thermal relaxation time, which guarantees that Eq. (6) will expect a limited speed of heat spread. The values of $\lambda$ and $\mu$ are defined as ${ }^{[34]}$

$$
\lambda=\frac{E \nu}{(1+\nu)(1-2 \nu)}, \quad \mu=\frac{E}{2(1+\nu)} .
$$

\section{Mathematical model description}

In this model, we assume that the thermoelastic micro-beam properties are unstrained, unstressed, and clamped-clamped. Let the constants $\rho, \sigma_{0}$, and $A$ denote the density, the initial tension, and the area cross-sectional of the micro-beam, respectively. As shown in Fig. 1, the micro-beam length, width, and uniform thickness are denoted as $L, b$, and $h$, respectively.

In order to formulate the governing equations of the thermoelastic micro-beam, the following assumptions are considered: (i) the cross-sectional area along the $x$-direction does not change; (ii) the Euler-Bernoulli beam theory is employed ${ }^{[35-36]}$; (iii) the only transverse motion is taken into consideration. As explained in Refs. [36]-[39], the axial motion can be very important and in some cases cannot be ignored. Based on the Euler-Bernoulli beam theory, the displacements may be written as follows:

$$
u=-z \frac{\partial w}{\partial x}, \quad v=0, \quad w=w(x, t)
$$




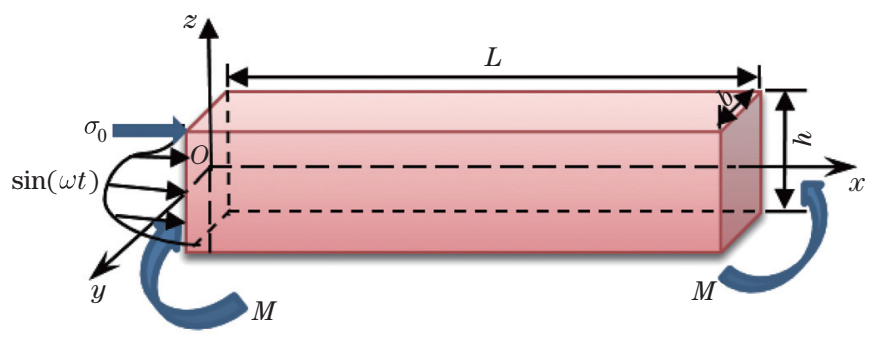

Fig. 1 Schematic diagram for the micro-beam (color online)

in which $u$ is the axial displacement and $w$ is the lateral deflection. By substituting Eq. (8) into Eq. (4), the rotation vector can be written as

$$
\omega_{y}=-\frac{\partial w}{\partial x}, \quad \omega_{x}=\omega_{z}=0 .
$$

The components of the curvature tensor are derived by substituting Eq. (9) into Eq. (3) as

$$
\chi_{x y}=-\frac{1}{2} \frac{\partial^{2} w}{\partial x^{2}}, \quad \chi_{x z}=\chi_{z x}=\chi_{z y}=\chi_{y z}=0 .
$$

Using Eq. (8), the axial thermal stress $\sigma_{x}$ given in Eq. (1) is reduced to

$$
\sigma_{x}=-E\left(z \frac{\partial^{2} w}{\partial x^{2}}+\alpha_{T} \theta\right) .
$$

The bending moment $M$ resultant of the micro-beam can be defined as follows:

$$
M=b \int_{-h / 2}^{h / 2} m_{x y} \mathrm{~d} z+b \int_{-h / 2}^{h / 2} z \sigma_{x} \mathrm{~d} z .
$$

Substituting Eqs. (2), (10), and (11) into Eq. (12), the moment $M$ is given by

$$
M(x, t)=-E I\left(\frac{\partial^{2} w}{\partial x^{2}}+\alpha_{T} M_{T}\right)-\alpha A \frac{\partial^{2} w}{\partial x^{2}},
$$

where

$$
M_{T}=\frac{12}{h^{3}} \int_{-h / 2}^{h / 2} \theta(x, z, t) z \mathrm{~d} z, \quad m_{x y}=-\frac{1}{2} \alpha \frac{\partial^{2} w}{\partial t^{2}} .
$$

The transversely governing equation of motion due to the initial compression stress $\sigma_{0}$ can be formulated as ${ }^{[30,40]}$

$$
\frac{\partial^{2} M}{\partial x^{2}}=\sigma_{0} A \frac{\partial^{2} w}{\partial x^{2}}+\rho A \frac{\partial^{2} w}{\partial t^{2}}
$$

Note that a negative estimation of $\sigma_{0}$ suggests the initial tensile stress. By substituting Eq. (13) into Eq. (15), the equation of motion can be expressed as

$$
\left((E I+\alpha A) \frac{\partial^{2}}{\partial x^{4}}+\rho A \frac{\partial^{2}}{\partial t^{2}}+\sigma_{0} A\right) w+\alpha_{T} E I \frac{\partial^{2} M_{T}}{\partial x^{2}}=0 .
$$

It can be noticed from Eq. (16) that the motion equation of the Euler-Bernoulli beam is composed of some parts: some of them are associated with $\rho A, \sigma_{0} A, E I$, and $\alpha A$, and the 
others are relevant to $\alpha_{T} E I$. The first part is the same as the one in the classical model, while the second part is added due to the existence of the MCS theory. The third part is due to the initial stress, and the last part is due to the temperature field. When the parameter of the couple stress of the material vanishes $(\alpha=0)$, the basic equation reduces to the classical model.

Substituting Eq. (8) into Eq. (6), the heat conduction will be in the form

$$
\frac{\partial^{2} \theta}{\partial x^{2}}+\frac{\partial^{2} \theta}{\partial z^{2}}=\left(1+\tau_{0} \frac{\partial}{\partial t}\right)\left(\frac{1}{k} \frac{\partial \theta}{\partial t}-\frac{\gamma T_{0} z}{K_{0}} \frac{\partial}{\partial t}\left(\frac{\partial^{2} w}{\partial x^{2}}\right)\right)
$$

The temperature $\theta(x, z, t)$ can be assumed to be in the form of sinusoidal function ${ }^{[41]}$, i.e.,

$$
\theta(x, z, t)=\Theta(x, t) \sin \left(\frac{\pi z}{h}\right)
$$

By substituting Eq. (18) into Eqs. (16) and (13), we get

$$
\begin{aligned}
& (E I+\alpha A) \frac{\partial^{2} w}{\partial x^{4}}+\rho A \frac{\partial^{2} w}{\partial t^{2}}+\sigma_{0} A \frac{\partial^{2} w}{\partial x^{2}}+E I \frac{24 \alpha_{T}}{\pi^{2} h} \frac{\partial^{2} \Theta}{\partial x^{2}}=0 \\
& M(x, t)=-(E I+\alpha A) \frac{\partial^{2} w}{\partial x^{2}}-E I \frac{24 \alpha_{T}}{\pi^{2} h} \Theta .
\end{aligned}
$$

Integrating Eq. (17) along the $z$-axis, the following heat equation is attained:

$$
\frac{\partial^{2} \Theta}{\partial x^{2}}-\frac{\pi^{2}}{h^{2}} \Theta=\left(1+\tau_{0} \frac{\partial}{\partial t}\right)\left(\frac{1}{k} \frac{\partial \Theta}{\partial t}-\frac{\gamma T_{0} \pi^{2} h}{24 K_{0}} \frac{\partial}{\partial t}\left(\frac{\partial^{2} w}{\partial x^{2}}\right)\right) .
$$

We use the non-dimensional quantities as follows:

$$
\left\{\begin{array}{l}
\left(x^{\prime}, u^{\prime}, w^{\prime}, z^{\prime}\right)=\frac{1}{L}(x, u, w, z), \quad \Theta^{\prime}=\frac{\gamma \Theta}{E}, \quad \sigma_{x}^{\prime}=\frac{\sigma_{x}}{E}, \\
\left(t^{\prime}, t_{0}^{\prime}\right)=\frac{c}{L}\left(t, t_{0}\right), \quad M^{\prime}=\frac{M}{b E h^{2}}, \quad c=\sqrt{\frac{E}{\rho}} .
\end{array}\right.
$$

The non-dimensional basic equations can be rewritten as (dropping primes for convenience)

$$
\begin{aligned}
& \frac{\partial^{4} w}{\partial x^{4}}+A_{1} \frac{\partial^{2} w}{\partial t^{2}}+A_{8} \frac{\partial^{2} w}{\partial x^{2}}+A_{2} \frac{\partial^{2} \Theta}{\partial x^{2}}=0 \\
& \frac{\partial^{2} \Theta}{\partial x^{2}}-A_{3} \Theta=\left(1+\tau_{0} \frac{\partial}{\partial t}\right)\left(A_{4} \frac{\partial \Theta}{\partial t}-A_{5} \frac{\partial}{\partial t}\left(\frac{\partial^{2} w}{\partial x^{2}}\right)\right), \\
& \sigma_{x}=-z \frac{\partial^{2} w}{\partial x^{2}}-\theta \\
& M(x, t)=-A_{6} \frac{\partial^{2} w}{\partial x^{2}}-A_{7} \Theta
\end{aligned}
$$

where

$$
\left\{\begin{array}{l}
A_{1}=\frac{L^{2} A E c^{2}}{E I+\alpha A}, \quad A_{2}=\frac{24 L E I}{\pi^{2} h(E I+\alpha A)}, \quad A_{3}=\frac{\pi^{2} L^{2}}{h^{2}}, \quad A_{4}=\frac{c L}{k} \\
A_{5}=\frac{\gamma^{2} T_{0} \pi^{2} h}{24 K E}, \quad A_{6}=\frac{(E I+\alpha A)}{b E h^{2} L}, \quad A_{7}=\frac{24 I \alpha_{T}}{\pi^{2} h b}, \quad A_{8}=\frac{L^{2} A \sigma_{0} E c^{2}}{E I+\alpha A} .
\end{array}\right.
$$




\section{Initial and boundary conditions}

The initial conditions are assumed to be homogeneous, i.e.,

$$
w(x, 0)=\frac{\partial w(x, 0)}{\partial t}=0, \quad \Theta(x, 0)=\frac{\partial \Theta(x, 0)}{\partial t}=0 .
$$

We consider the case that the two ends of the micro-beam are clamped. Then, the following boundary conditions are expressed as:

$$
\left.w(x, t)\right|_{x=0, L}=0,\left.\quad \frac{\partial w(x, t)}{\partial x}\right|_{x=0, L}=0 .
$$

Also, we suppose that the surface $x=0$ of a micro-beam is subject to a temperature pulse in the form

$$
\Theta(0, t)=\Theta_{0}\left\{\begin{array}{l}
\sin (\omega t), \quad 0 \leqslant t \leqslant \frac{\pi}{\omega}, \\
0, \quad t>\frac{\pi}{\omega},
\end{array}\right.
$$

where $\Theta_{0}$ is the amplitude of the thermal load, and $\omega$ is the temperature pulse. Furthermore, the temperature change at the end boundary $x=L$ should satisfy the condition

$$
\frac{\partial \Theta}{\partial x}=0, \quad x=L .
$$

\section{Solution in the transformed domain}

The Laplace transform is characterized by the integral

$$
\bar{f}(x, s)=\int_{0}^{\infty} \mathrm{e}^{-s t} f(x, t) \mathrm{d} t .
$$

The governing equations in the Laplace domain are given by

$$
\begin{aligned}
& \frac{\mathrm{d}^{4} \bar{w}}{\mathrm{~d} x^{4}}+A_{8} \frac{\mathrm{d}^{2} \bar{w}}{\mathrm{~d} x^{2}}+A_{1} s^{2} \bar{w}+A_{2} \frac{\mathrm{d}^{2} \bar{\Theta}}{\mathrm{d} x^{2}}=0, \\
& \left(\frac{\mathrm{d}^{2}}{\mathrm{~d} x^{2}}-B_{1}\right) \bar{\Theta}=-B_{2} \frac{\mathrm{d}^{2} \bar{w}}{\mathrm{~d} x^{2}}, \\
& \bar{\sigma}_{x}=-z \frac{\mathrm{d}^{2} \bar{w}}{\mathrm{~d} x^{2}}-\bar{\theta} \\
& \bar{M}=-A_{6} \frac{\mathrm{d}^{2} \bar{w}}{\mathrm{~d} x^{2}}-A_{7} \bar{\Theta}
\end{aligned}
$$

where

$$
B_{1}=A_{3}+s\left(1+\tau_{0} s\right) A_{4}, \quad B_{2}=s\left(1+\tau_{0} s\right) A_{5}
$$

Combining Eq. (33) and Eq. (34) gives the differential equation for $\bar{w}$ or $\bar{\Theta}$ as

$$
\left(\frac{\mathrm{d}^{6}}{\mathrm{~d} x^{6}}-A \frac{\mathrm{d}^{4}}{\mathrm{~d} x^{4}}+B \frac{\mathrm{d}^{2}}{\mathrm{~d} x^{2}}-C\right)\{\bar{w}, \bar{\Theta}\}=0
$$

where the coefficients $A, B$, and $C$ are given, respectively, by

$$
A=B_{1}+A_{2} B_{2}-A_{8}, \quad B=s^{2} A_{1}-B_{1} A_{8}, \quad C=A_{1} B_{1} s^{2} .
$$


Introducing $m_{i}$ into Eq. (38) yields

$$
\left(\mathrm{D}^{2}-m_{1}^{2}\right)\left(\mathrm{D}^{2}-m_{2}^{2}\right)\left(\mathrm{D}^{2}-m_{3}^{2}\right)\{\bar{w}, \bar{\Theta}\}=0,
$$

where $\mathrm{D}=\mathrm{d} / \mathrm{d} x$, and $m_{1}^{2}, m_{2}^{2}$, and $m_{3}^{2}$ are the characteristic roots of the equation

$$
m^{6}-A m^{4}+B m^{2}+C=0 .
$$

The roots of Eq. (41) achieve the notable relations

$$
m_{1}^{2}+m_{2}^{2}+m_{2}^{2}=A, \quad m_{1}^{2} m_{2}^{2}+m_{2}^{2} m_{3}^{2}+m_{3}^{2} m_{1}^{2}=B, \quad m_{1}^{2} m_{2}^{2} m_{2}^{2}=C .
$$

The analytical solution to Eq. (40) can be calculated as

$$
\begin{aligned}
& \bar{w}=\sum_{i=1}^{3}\left(L_{i} \cosh \left(m_{i} x\right)+M_{i} \sinh \left(m_{i} x\right)\right) \\
& \bar{\Theta}=\sum_{i=1}^{3} \beta_{i}\left(L_{i} \cosh \left(m_{i} x\right)+M_{i} \sinh \left(m_{i} x\right)\right),
\end{aligned}
$$

where $L_{i}$ and $M_{i}$ are constant coefficients depending on $s$, and

$$
\beta_{i}=-\frac{B_{2} m_{i}^{2}}{m_{i}^{2}-B_{1}}
$$

The bending moment $\bar{M}$, given in Eq. (36) in the Laplace space using Eqs. (43) and (44), can be gained as

$$
\bar{M}=-\sum_{i=1}^{3} \gamma_{i}\left(L_{i} \cosh \left(m_{i} x\right)+M_{i} \sinh \left(m_{i} x\right)\right)
$$

where $\gamma_{i}=A_{6} m_{i}^{2}+A_{7} \beta_{i}$.

Also, the axial displacement after using Eq. (43) takes the form

$$
\bar{u}=-z \frac{\mathrm{d} \bar{w}}{\mathrm{~d} x}=z \sum_{i=1}^{3} m_{i}\left(L_{i} \sinh \left(m_{i} x\right)+M_{i} \cosh \left(m_{i} x\right)\right) .
$$

Furthermore, the strain can be calculated as

$$
\begin{aligned}
& \bar{e}=\frac{\mathrm{d} \bar{u}}{\mathrm{~d} x}=-z \sum_{i=1}^{3} m_{i}^{2}\left(L_{i} \cosh \left(m_{i} x\right)+M_{i} \sinh \left(m_{i} x\right)\right), \\
& \bar{\sigma}_{x}=-\sum_{i=1}^{3}\left(z m_{i}^{2}+\beta_{i} \sin (\pi z / h)\right)\left(L_{i} \cosh \left(m_{i} x\right)+M_{i} \sinh \left(m_{i} x\right)\right) .
\end{aligned}
$$

In the domain of the Laplace transform, the boundary conditions (29)-(31) take the forms

$$
\begin{aligned}
& \left.\bar{w}(x, s)\right|_{x=0, L}=0,\left.\quad \frac{\mathrm{d} \bar{w}(x, s)}{\mathrm{d} x}\right|_{x=0, L}=0, \\
& \left.\bar{\Theta}(x, s)\right|_{x=0}=\frac{\omega \Theta_{0}}{s^{2}+\omega^{2}}=\bar{G}(s) . \\
& \frac{\partial \bar{\Theta}}{\partial x}=0, \quad x=L .
\end{aligned}
$$


Substituting Eqs. (50)-(52) into Eqs. (43) and (44) yields

$$
\begin{aligned}
& \sum_{i=1}^{3} L_{i}=0 \\
& \sum_{i=1}^{3}\left(L_{i} \cosh \left(m_{i} L\right)+M_{i} \sinh \left(m_{i} L\right)\right)=0 \\
& \sum_{i=1}^{3} m_{i} M_{i}=0 \\
& \sum_{i=1}^{3} m_{i}\left(L_{i} \sinh \left(m_{i} L\right)+M_{i} \cosh \left(m_{i} L\right)\right)=0 \\
& \sum_{i=1}^{3} \beta_{i} L_{i}=\bar{G}(s) \\
& \sum_{i=1}^{3} \beta_{i} m_{i}\left(L_{i} \sinh \left(m_{i} L\right)+M_{i} \cosh \left(m_{i} L\right)\right)=0 .
\end{aligned}
$$

To complete the analytical solution within the Laplace transform domain, it remains for us to find the values of the coefficients $L_{i}$ and $M_{i}$. These values can be easily derived by solving the system of equations (53)-(58).

To obtain the field variables in the physical domain, the inversion of the Laplace transform will be derived. Due to the complexity of obtaining the inversion of the Laplace transform analytically, instead, the Riemann-sum approximation technique will be employed. To accomplish this task, the following well-known Riemann-sum approximation formula defined will be used $^{[42]}$ :

$$
f(x, z, t)=\frac{\mathrm{e}^{v t}}{t}\left(\frac{1}{2} \operatorname{Re}(\bar{F}(x, z, v))+\operatorname{Re} \sum_{n=0}^{N}\left(\bar{F}\left(x, z, v+\frac{i n \pi}{t}\right)(-1)^{n}\right)\right) .
$$

For convergence of the solution reasons, the Riemann-sum approximation technique ${ }^{[43]}$ insists that $v \approx 4.7 / t$.

\section{Numerical results}

In this section, the effects of the temperature pulse, the absence and presence of the couple stress, and the relaxation time on the field amounts are numerically discussed. All the numerical calculations are conducted using MATHEMATICA platform. The mechanical properties of the Nickel micro-beam can be introduced as ${ }^{[44]}$

$$
\left\{\begin{array}{l}
E=210 \mathrm{GPa}, \quad \rho=8900 \mathrm{~kg} / \mathrm{m}^{3}, \quad C_{E}=438 \mathrm{~J} /(\mathrm{kg} \cdot \mathrm{K}), \\
\alpha_{T}=13 \times 10^{-6} \mathrm{~K}^{-1}, \quad \nu=0.31, \quad K=92 \mathrm{~W} /(\mathrm{m} \cdot \mathrm{K}) .
\end{array}\right.
$$

We assume that the values of the micro-beam non-dimensional operational parameters are assigned as $h=10, L / h=10, b / h=0.5, t=0.12 \mathrm{~s}$, and $\Theta_{0}=1$. Using the approximation method defined in Eq. (59), the values of the deflection $w$, temperature $\theta$, thermal moment $M$, displacement $u$, and stress $\sigma_{x}$ are computed. The effects of the presence of the couple stress, initial stress, and temperature pulse on the micro-beam will be introduced.

\subsection{Couple stress effect}

This subsection indicates the effects of the small length scale $\alpha$ (couple stress) on microbeams in terms of the deflection $w$, temperature $\theta$, thermal moment $M$, displacement $u$, and 
stress $\sigma_{x}$ in two cases. Namely, the presence and absence of couple stress are indicated as $\alpha=2.5$ and 0.0 , respectively. Figures $2-6$ illustrate the two cases, where the parameters $\sigma_{0}, \omega$, and $\tau_{0}$ are fixed to certain values.

In order to verify the results obtained in this subsection, they are compared with the results of the classical Euler-Bernoulli beam. It is worth noting that the values obtained by the MCS theory in the present study are always greater than the calculated values based on the classical theory. This important observation is consistent with the results obtained by Babaei et al. ${ }^{[45]}$.

As displayed in Fig. 2, compared with the classical theory considering the effect of the material length scale parameter on the MCS theory, it leads to a decrease in the lateral deflection of the micro-beam. Therefore, taking into account the parameter of the material length scale, the maximum and minimum of the lateral deflection decrease. Also, as time goes by, the deflection starts from zero and reaches a maximum value at $x=0.2$ based on the couple stress theory, and finally quickly tends to zero, satisfying the mechanical boundary condition.

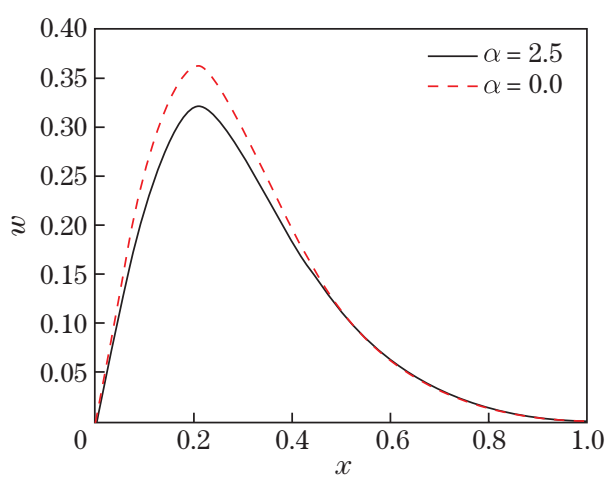

Fig. 2 The transverse deflection $w$ versus $x$ with different modified couple stress parameters $\alpha$ (color online)

Figure 3 depicts that the parameter $\alpha$ has a small effect on the temperature change in both the MCS and the classical theory models, where the two curves are very close to each other. On the other hand, the figure indicates that the temperature values drop dramatically in the micro-beam towards the length of the beam. This situation corresponds to the physical state.

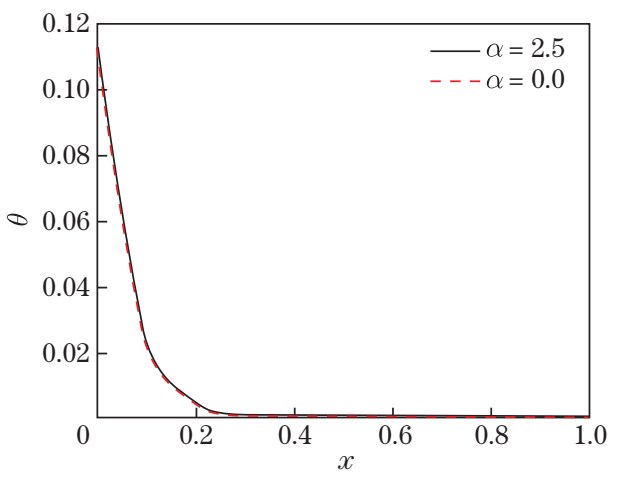

Fig. 3 The temperature $\theta$ versus $x$ with different modified couple stress parameters $\alpha$ (color online)

Thus, we can conclude that the thermal vibrations of the micro-beams obtained with the help of the MCS are higher than those predicted by the classical theory of Euler-Bernoulli beams. Comparing the results with Kong et al. ${ }^{[46]}$, we find that there is a convergence of the results. 
As can be seen in Fig. 4, the MCS model predicts lower values of natural axial stress $\sigma_{x}$ in comparison with the classical theory. The parameter $\alpha$ has a significant effect on the distribution of the bending moment $M$ as shown in Fig. 5. It is clearly observed in Fig. 5 that the value of the bending moment $M$ decreases with a decrease in the value of the small length scale parameter $\alpha$, and this corresponds to Refs. [47]-[49]. Figure 6 illustrates that the value of the axial displacement $u$ goes down in the range $0 \leqslant x \leqslant 0.1$ and then goes up to the maximum amplitudes in the range $0.1 \leqslant x \leqslant 0.3$. In addition, the small length scale parameter $\alpha$ has a great impact on the displacement distribution $u$. From Figs. 2-6, we note that the values of distribution fields increase in the presence of the couple stress term and decreases in the absence of that term.

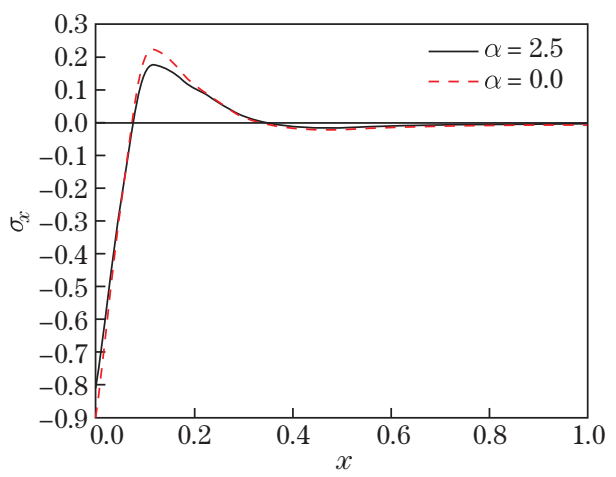

Fig. 4 The stress $\sigma_{x}$ versus $x$ with different modified couple stress parameters $\alpha$ (color online)

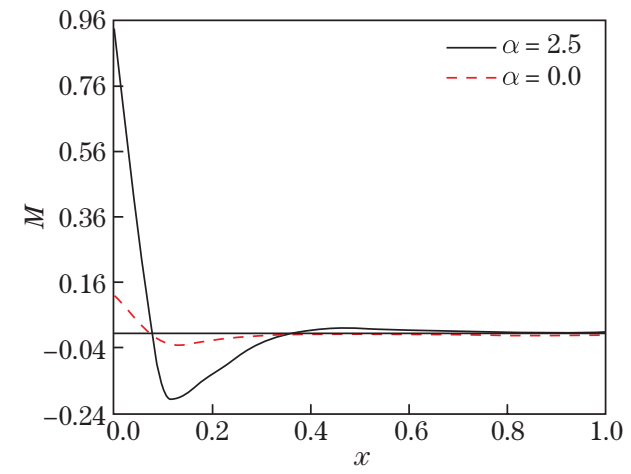

Fig. 5 The bending moment $M$ versus $x$ with different modified couple stress parameters $\alpha$ (color online)

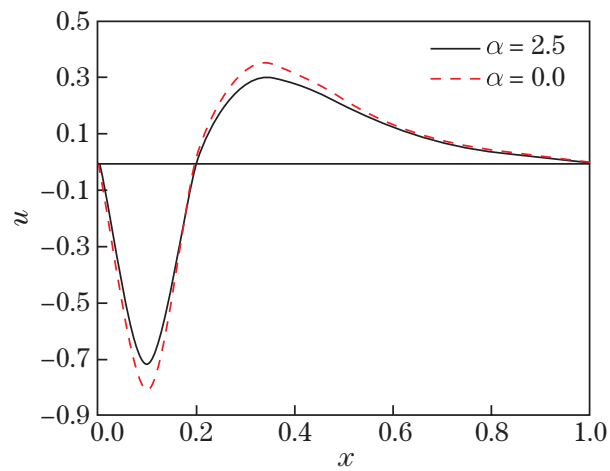

Fig. 6 The displacement $u$ versus $x$ with different modified couple stress parameters $\alpha$ (color online)

\subsection{The initial stress effect on solid materials}

This case depicts the variety of the dimensionless physical fields of the micro-beam with three different values of the dimensionless initial stress $\sigma_{0}$ (see Figs. 7-11). In this case, we have assumed that the pulse of temperature $\omega=0.2$ and the relaxation time $\tau_{0}=0.1$. Note that, the value of $\sigma_{0}=0$ indicates the absence of the initial stress, while other values indicate that the micro-beam is subject to compressive strength.

Figure 7 shows the deflection of the micro-beam for various values of the non-dimensional initial stress parameter $\sigma_{0}$. Obviously, by raising the value of the parameter $\sigma_{0}$, the deflection increases. Figure 8 displays the temperature response of the micro-beam for various values of the initial stress parameter versus the distance $x$. In this figure, the increase in the initial stress force $\sigma_{0}$ causes a weak effect on the temperature of the micro-beam. Figures 9-11 indicate the 


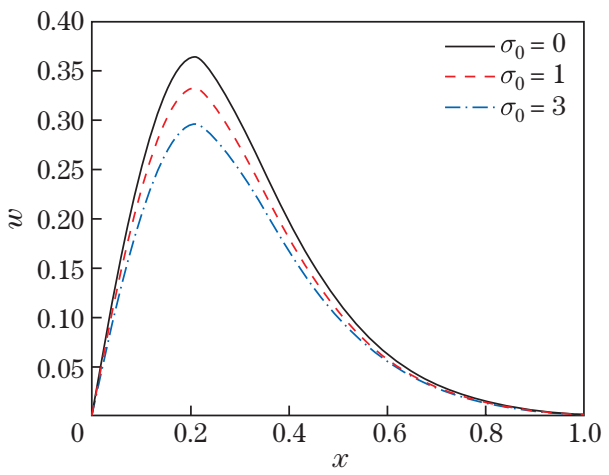

Fig. 7 The transverse deflection $w$ versus $x$ with different initial stress parameters $\sigma_{0}$ (color online)

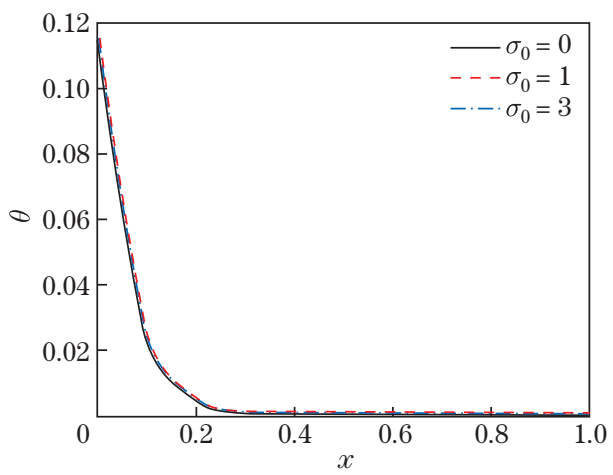

Fig. 8 The temperature $\theta$ versus $x$ with different initial stress parameters $\sigma_{0}$ (color online)

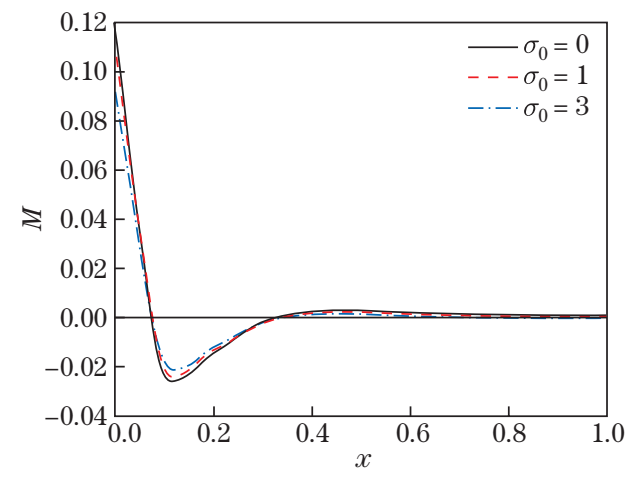

Fig. 10 The bending moment $M$ versus $x$ with different initial stress parameters $\sigma_{0}$ (color online)

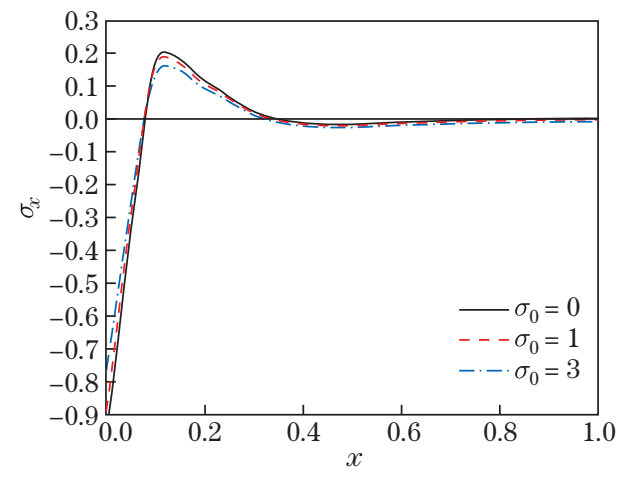

Fig. 9 The stress $\sigma_{x}$ versus $x$ with different initial stress parameters $\sigma_{0}$ (color online)

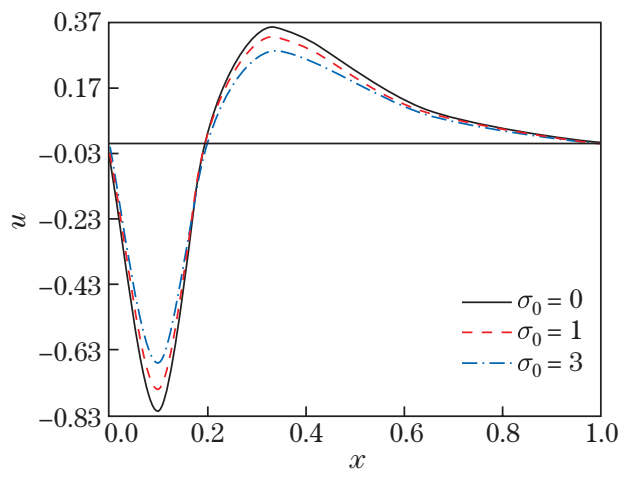

Fig. 11 The displacement $u$ versus $x$ with different initial stress parameters $\sigma_{0}$ (color online)

variations of the thermal stress $\sigma_{x}$, the displacement $u$, and the bending moment $M$ for various values of the dimensionless initial stress $\sigma_{0}$. As can be seen, the initial pressure increase leads to an increase in the field variables.

\subsection{The effect of temperature pulse}

Figures 12-16 display the reactions of a clamped-clamped micro-beam resonator in the direction of the axial $x$ with different temperature pulses $\omega, \sigma_{0}=1$, and $\alpha=2.5$. 


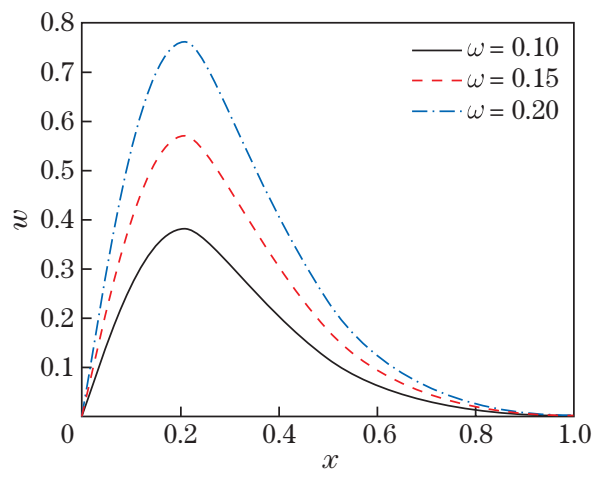

Fig. 12 The transverse deflection $w$ versus $x$ with different temperature pulses $\omega$ (color online)

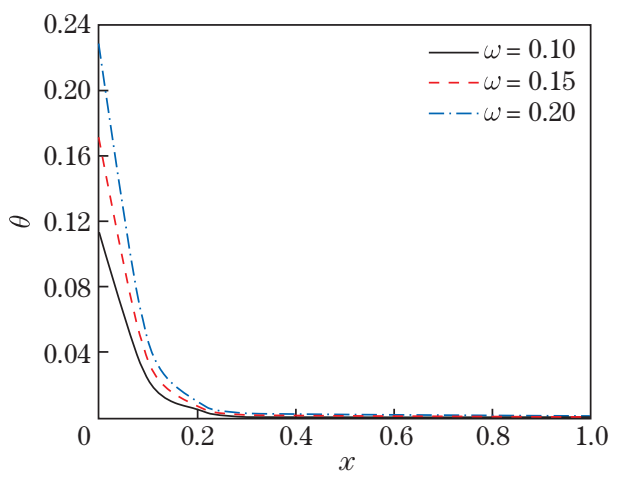

Fig. 13 The temperature $\theta$ versus $x$ with different temperature pulses $\omega$ (color online)

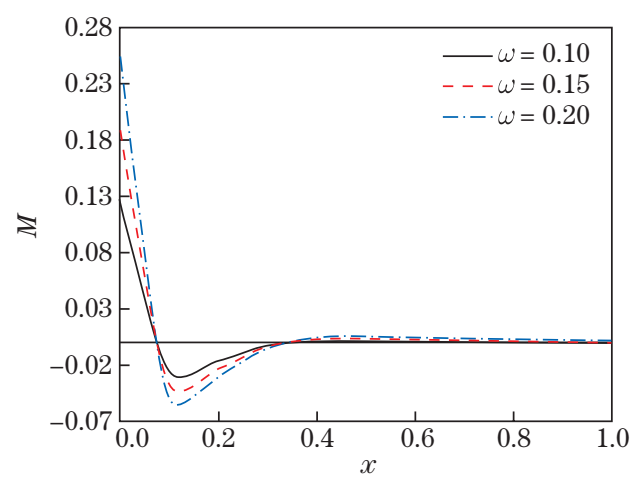

Fig. 15 The bending moment $M$ versus $x$ with different temperature pulses $\omega$ (color online)

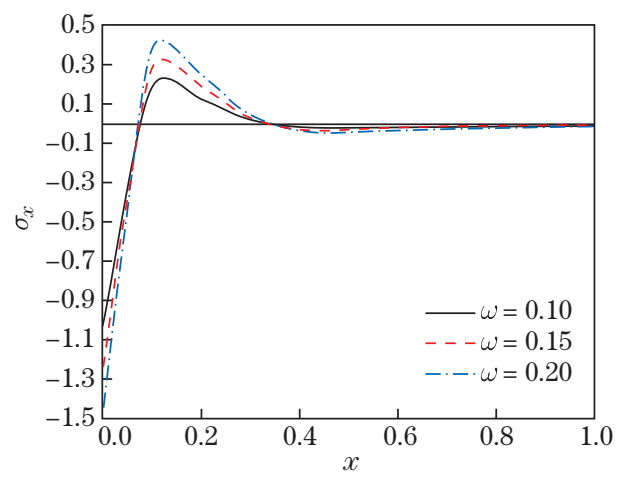

Fig. 14 The stress $\sigma_{x}$ versus $x$ with different temperature pulses $\omega$ (color online)

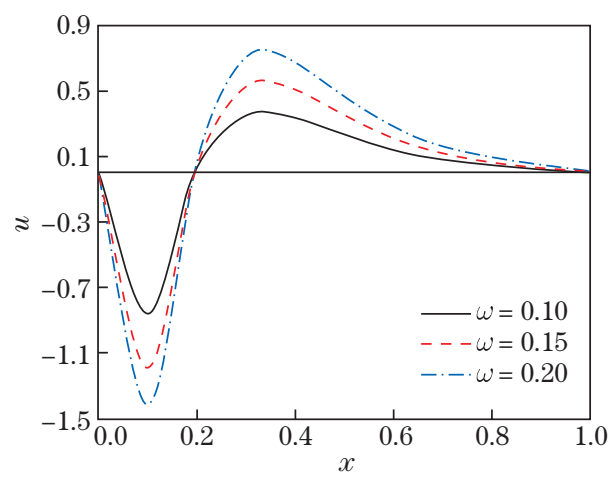

Fig. 16 The displacement $u$ versus $x$ with different temperature pulses $\omega$ (color online)

From these figures, we note that the pulse temperature constant has a significant effect on the mechanical behaviors of the Euler-Bernoulli micro-beam. The inclusion of the temperature pulse effect in the beam theory leads to an increase in the thermodynamic interactions in the micro-beam. As a result, this study gives a physical realization that can be practical to design and analyze the vibrations for micro-/nano-structures. 


\subsection{Comparable results in Lord-Shulman (LS) and coupled thermoelasticity (CTE) theories}

In the current case, the responses of variable fields in the cases of the theories of coupled thermoelasticity and the Lord-Shulman theory are analyzed. The calculations are conducted based on the values of the relaxation parameter $\tau_{0}$. If $\tau_{0}=0$, the responses of variable fields are applied to the CTE theory. If $\tau_{0}>0$, the responses of variable fields are applied to the LS theory. The other parameters are assumed to be constants. From Figs. 17-21, we conclude that: (i) the magnitudes of the considered field variables in the CTE model are greater compared with those in the LS model; (ii) the parameter $\tau_{0}$ has a great effect on the propagation of all field quantities; (iii) the micro-beam exhibits more deflection in the case of the coupled thermoelastic (CT) beam than that for the generalized thermoelastic LS model; (iv) the mechanical distributions indicate that the wave propagates with a finite velocity in the medium; (v) although the thermal wave spreads with a finite speed in the coupled theory thermoelasticity as seen in Figs. 17-21, there are great differences between the coupled theory and the generalized theory.

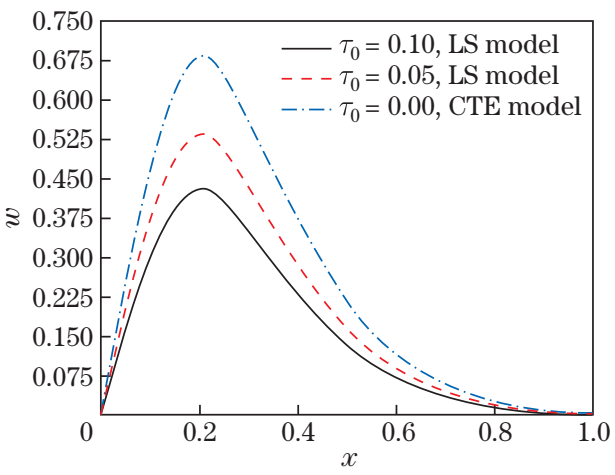

Fig. 17 The transverse deflection $w$ versus $x$ for different theories of thermoelasticity (color online)

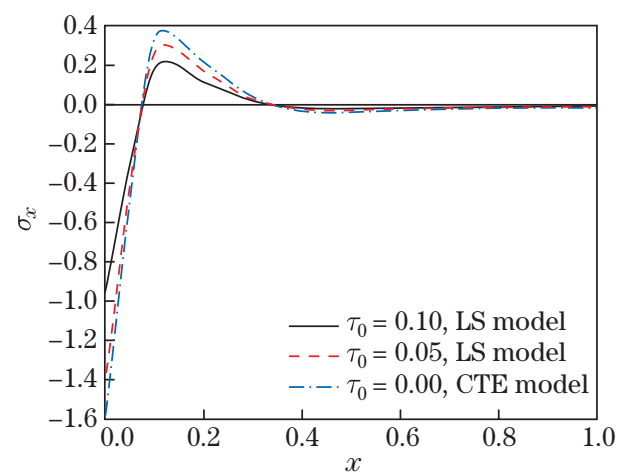

Fig. 19 The stress $\sigma_{x}$ versus $x$ for different theories of thermoelasticity (color online)

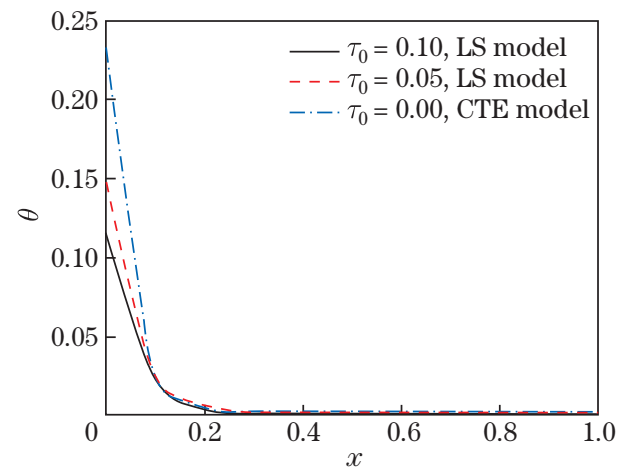

Fig. 18 The temperature $\theta$ versus $x$ for different theories of thermoelasticity (color online)

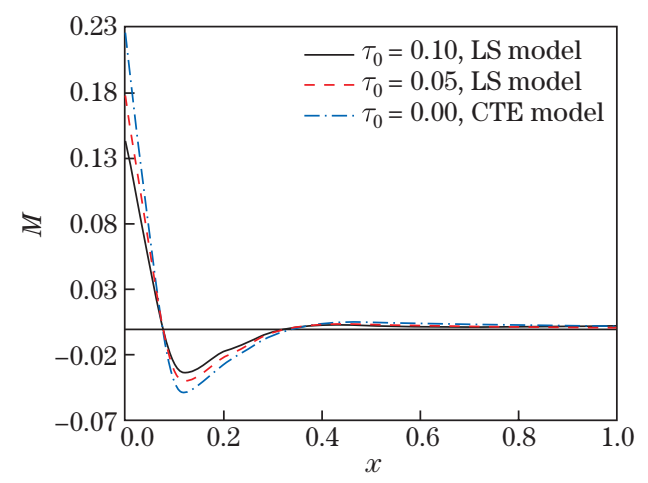

Fig. 20 The bending moment $M$ versus $x$ for different theories of thermoelasticity (color online)

\section{Conclusions}

In this work, the effects of the initial stress, temperature pulse heating, and MCS term on the thermoelastic response of a micro-beam are mathematically analyzed. A governing equation 


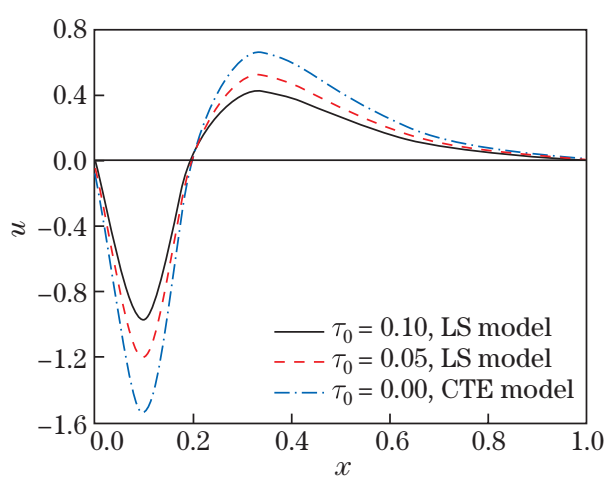

Fig. 21 The displacement $u$ versus $x$ for different theories of thermoelasticity (color online)

that governs the studied problem dependent on the Euler-Bernoulli theory is proposed by the use of Hamiltonian's principle. Using the Laplace transform, the expressions of the field variables are derived. The proposed model is numerically analyzed using MATHEMATICA.

The numerical results indicate that the parameter of the couple stress has significant effects on all the distributions of the studied fields. Increasing the effect of couple stress leads to decreasing the values of the basic field variables. Furthermore, the thermoelastic deflections, thermal stress, displacement, temperature, and moment strongly depend on the pulse of temperature.

The current investigation can be furthered to the analysis and design of micro-structures with geometrical shapes and different load conditions under different boundary conditions based on the Euler-Bernoulli beam theory, and the obtained results may be valuable to mechanical engineers in designing small scale resonators and MEMS applications.

Open Access This article is licensed under a Creative Commons Attribution 4.0 International License, which permits use, sharing, adaptation, distribution and reproduction in any medium or format, as long as you give appropriate credit to the original author (s) and the source, provide a link to the Creative Commons licence, and indicate if changes were made. To view a copy of this licence, visit http://creativecommons.org/licenses/by/4.0/.

\section{References}

[1] YANG, F., CHONG, A. C. M., LAM, D. C. C., and TONG, P. Couple stress based strain gradient theory for elasticity. International Journal of Solids and Structures, 39, 2731-2743 (2002)

[2] AKGOZ, B. and CIVALEK, O. Strain gradient elasticity and modified couple stress models for buckling analysis of axially loaded micro-scaled beams. International Journal of Engineering Science, 49, 1268-1280 (2011)

[3] REDDY, J. N. and KIM J. A nonlinear modified couple stress-based third-order theory of functionally graded plates. Composite Structures, 94, 1128-1143 (2012)

[4] ABOUELREGAL, A. E. and MOHAMMED, W. W. Effects of nonlocal thermoelasticity on nanoscale beams based on couple stress theory. Mathematical Methods in the Applied Sciences (2020) https://doi.org/10.1002/mma.6764

[5] STÖLKEN, J. and EVANS, A. A microbend test method for measuring the plasticity length scale. Acta Materialia, 46, 5109-5115 (1998)

[6] CHONG, A. and LAM, D. C. Strain gradient plasticity effect in indentation hardness of polymers. Journal of Materials Research, 14, 4103-4110 (1999)

[7] MCFARLAND, A. W. and COLTON, J. S. Role of material microstructure in plate stiffness with relevance to microcantilever sensors. Journal of Micromechanics and Microengineering, 15, 1060-1067 (2005) 
[8] ABOUELREGAL, A. E. Response of thermoelastic micro-beams to a periodic external transverse excitation basedon MCS theory. Microsystem Technologies, 24, 1925-1933 (2018)

[9] FAROKHI, H. and GHAYESH, M. H. Viscoelastic shear deformable microplates: nonlinear forced resonant characteristics. Mechanical Systems and Signal Processing, 118, 742-756 (2019)

[10] ZHANG, N., ZHENG, S., and CHEN, D. Size-dependent static bending of flexomagnetic nanobeams. Journal of Applied Physics, 126, 223901 (2019)

[11] ABOUELREGAL, A. E. A novel model of nonlocal thermoelasticity with time derivatives of higher order. Mathematical Methods in the Applied Sciences, 43, 6746-6760 (2020)

[12] ALIBEIGI, B. and TADI BENI, Y. On the size-dependent magneto/electromechanical buckling of nanobeams. The European Physical Journal Plus, 133, 398 (2018)

[13] MINDLIN, R. and TIERSTEN, H. Effects of couple-stresses in linear elasticity. Archive for Rational Mechanics and Analysis, 11, 415-448 (1962)

[14] TOUPIN, R. A. Elastic materials with couple-stresses. Archive for Rational Mechanics and Analysis, 11, 385-414 (1962)

[15] SIDHARDH, S. and RAY, M. C. Size-dependent elastic response in functionally graded microbeams considering generalized first strain gradient elasticity. The Quarterly Journal of Mechanics and Applied Mathematics, 72, 273-304 (2019)

[16] KUMAR, R. Response of thermoelastic beam due to thermal source in modified couple stress theory. Computational Methods in Science and Technology, 22, 95-101 (2016)

[17] PARK, S. K. and GAO, X. L. Bernoulli-Euler beam model based on a modified couple stress theory. Journal of Micromechanics and Microengineering, 16, 2355-2359 (2006)

[18] QU, Y., LI, P., and JIN, F. A general dynamic theoretical model of elastic micro-structures with consideration of couple stress effects and its application in mechanical analysis of size-dependent properties. Acta Mechanica, 231, 471-488 (2020)

[19] WANG, B., ZHAO, J., and ZHOU, S. A micro scale Timoshenko beam model based on strain gradient elasticity theory. European Journal of Mechanics-A/Solids, 29, 591-599 (2010)

[20] MINDLIN, R. D. and TIERSTEN, H. F. Effects of couple-stresses in linear elasticity. Archive for Rational Mechanics and Analysis, 11, 415-448 (1962)

[21] YANG, F., CHONG, A. C. M., LAM, D. C. C., and TONG, P. Couple stress based strain gradient theory for elasticity. International Journal of Solids and Structures, 39, 2731-2743 (2002)

[22] TSIATAS, G. C. A new Kirchhoff plate model based on a modified couple stress theory. International Journal of Solids and Structures, 46, 2757-2764 (2009)

[23] WANG, L., CHEN, H. H., and HE, X. D. Active $H_{\infty}$ control of the vibration of an axially moving cantilever beam by magnetic force. Mechanical Systems and Signal Processing, 25, 2863-2878 (2011)

[24] DUAN, Y. C., WANG, J. P., SHAO, F., and LIU, Y. W. Theoretical and experimental study on the dynamic characteristics of an axially moving nested clamped-hinged beam. Journal of Vibroengineering, 17, 602-619 (2015)

[25] MA, H. M., GAO, X. L., and REDDY, J. N. A microstructure dependent Timoshenko beam model based on a modified couple stress theory. Journal of the Mechanics and Physics of Solids, 56, 3379-3391 (2008)

[26] GHAYESH, M. H., FAROKHI, H., and AMABILI, M. Nonlinear dynamics of a microscale beam based on the modified couple stress theory. Composites Part B: Engineering, 50, 318-324 (2013)

[27] ABO-DAHAB, S. M., ABOUELREGAL, A. E., and MARIN M. Generalized thermoelastic functionally graded on a thin slim strip non-Gaussian laser beam. Symmetry, 12(7), 1094 (2020)

[28] GHAYESH, M. H. Dynamics of functionally graded viscoelastic micro-beams. International Journal of Engineering Science, 124, 115-131 (2018)

[29] CARrerA, E., ABOUElregAl, A. E., ABBAS, I. A., and ZENKOUR, A. M. Vibrational analysis for an axially moving micro-beam with two temperatures. Journal of Thermal Stresses, 38, 569-590 (2015)

[30] YAO, L. Q., JI, C. J., SHEN, J. P., and LI, C. Free vibration and wave propagation of axially moving functionally graded Timoshenko microbeams. Journal of the Brazilian Society of Mechanical Sciences and Engineering, 42, 173 (2020) 
[31] WANG, J., SHEN, H., ZHANG, B., LIU, J., and ZHANG, Y. Complex modal analysis of transverse free vibrations for axially moving nanobeams based on the nonlocal strain gradient theory. Physica $E$, 101, 85-93 (2018)

[32] HADJESFANDIARI, A. R. and DARGUSH, G. F. Couple stress theory for solids. International Journal of Solids and Structures, 48, 2496-2510 (2011)

[33] LORD, H. W. and SHULMAN, Y. A generalized dynamical theory of thermoelasticity. Journal of the Mechanics and Physics of Solids, 15, 299-309 (1967)

[34] SADD, M. H. Elasticity Theory Application and Numerics, Academic Press, Salt Lake City, UT (2009)

[35] BENHAMED, M. M. and ABOUELREGAL, A. E. Influence of temperature pulse on a nickel micro-beams under couple stress theory. Journal of Applied and Computational Mechanics, 6(4), 777-787 (2020)

[36] GHAYESH, M. H., FAROKHI, H., and HUSSAIN, S. Viscoelastically coupled size-dependent dynamics of micro-beams. International Journal of Engineering Science, 109, 243-255 (2016)

[37] SIDIG, A. and ABOUELREGAL, A. E. Thermoelastic response of micro-beams under a magnetic field rested on two-parameter viscoelastic foundation. Journal of Computational Applied Mechanics (2020) https://doi.org/10.22059/jcamech.2019.293933.460

[38] ABOUELREGAL, A. E. and ZENKOUR, A. M. Dynamic characteristics of initially stressed viscoelastic micro-beams induced by ultra-intense lasers. Indian Journal of Physics, 94, 779-788 (2020)

[39] GHAYESH, M. H., FAROKHI, H., and ALICI, G. Size-dependent performance of microgyroscopes. International Journal of Engineering Science, 100, 99-111 (2016)

[40] WANG, C. M., ZHANG, Y. Y., and KITIPORNCHAI, S. Vibration of initially stressed microand nano-beams. International Journal of Structural Stability and Dynamics, 7(4), 555-570 (2007)

[41] TAATI, E., NAJAFABADI, M. M., and TABRIZI, B. H. Size-dependent generalized thermoelasticity model for timoshenko micro-beams. Acta Mechanica, 225, 1823-1842 (2014)

[42] HONIG, G. and HIRDES, U. A method for the numerical inversion of the Laplace transform. Journal of Computational and Applied Mathematics, 10, 113-132 (1984)

[43] TZOU, Y. Experimental support for the lagging behavior in heat propagation. Journal of Thermophysics and Heat Transfer, 9(4), 686-693 (1995)

[44] NAJAFI, M., REZAZADEH, G., and SHABANI, R. Thermoelastic damping in a capacitive microbeam resonator considering hyperbolic heat conduction model and modified couple stress theory. Journal of Solid Mechanics, 4(4), 386-401 (2012)

[45] BABAEI, A., NOORANI, M. R. S., and GHANBARI, A. Temperature-dependent free vibration analysis of functionally graded micro-beams based on the modified couple stress theory. Microsystem Technologies, 23, 4599-4610 (2017)

[46] KONG, S., ZHOU, S., NIE, Z., and WANG, K. The size-dependent natural frequency of BernoulliEuler micro-beams. International Journal of Engineering Science, 46, 427-437 (2008)

[47] KUMAR, R. and DEVI, S. Eigenvalue approach to nanobeam in modified couple stress thermoelastic with three-phase-lag model induced by ramp type heating. Journal of Theoretical and Applied Mechanics, 55(3), 1067-1079 (2017)

[48] YANG, X. D., LIU, M., ZHANG, W., and MELNIK, R. V. N. Invariant and energy analysis of an axially retracting beam. Chinese Journal of Aeronautics, 29, 952-961 (2016)

[49] HAMIDI, B. A., HOSSEINI, S. A., HASSANNEJAD, R., and KHOSRAVI, F. An exact solution on gold micro-beam with thermoelastic damping via generalized Green-Naghdi and modified couple stress theories. Journal of Thermal Stresses, 34(2), 157-174 (2020) 